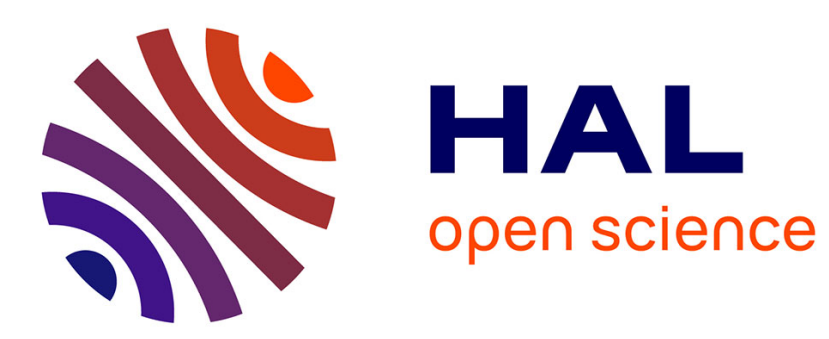

\title{
L'agritourisme dans les Alpes slovènes depuis 1991, choix ou contrainte pour les ménages agricoles?
}

\author{
Angela Barthes
}

\section{To cite this version:}

Angela Barthes. L'agritourisme dans les Alpes slovènes depuis 1991, choix ou contrainte pour les ménages agricoles?. Autrepart - Revue de sciences sociales au Sud, 2008, n²46, p143-156. halshs00388546

\section{HAL Id: halshs-00388546 \\ https://shs.hal.science/halshs-00388546}

Submitted on 26 May 2009

HAL is a multi-disciplinary open access archive for the deposit and dissemination of scientific research documents, whether they are published or not. The documents may come from teaching and research institutions in France or abroad, or from public or private research centers.
L'archive ouverte pluridisciplinaire HAL, est destinée au dépôt et à la diffusion de documents scientifiques de niveau recherche, publiés ou non, émanant des établissements d'enseignement et de recherche français ou étrangers, des laboratoires publics ou privés. 


\title{
L'agritourisme dans les Alpes slovènes depuis 1991, choix ou contrainte pour les ménages agricoles?
}

\author{
Barthes Angela, Maître de conférence de l'Université de Provence \\ UMR Telemme \\ abarthes@up.univ-mrs.fr
}

\section{Résumé}

L'effondrement productif de l'agriculture des régions alpines depuis 1991, la forte contraction des actifs agricoles en 15 ans (-60\%), les pertes d'emplois et de revenus, engendrent des difficultés de reconversion de l'espace montagnard. L'article se propose d'examiner la situation des ménages paysans, exploitants et propriétaires en 1991, et résidants toujours dans leur ferme en 2005. L'analyse diachronique détaillée entre 1991et 2005 de leur structure familiale et de leurs revenus, en regard des stratégies d'adaptation : abandon, diversification ou intensification des activités, amène à repenser les choix effectifs en termes de contraintes objectives. Par ailleurs, les rapports entre les évolutions des stratégies dominantes des ménages paysans et les types d'espaces montagnards apportent des indications sur les potentialités réelles de reconversion des exploitants vers l'agritourisme. La faible emprise du tourisme rural permet d'établir que, s'il concourt à une ouverture culturelle des espaces ruraux et constitue une des solutions de leur reconversion fonctionnelle, il est loin de remplacer les dynamiques économiques antérieures.

Mots clefs : Agritourisme, Alpes slovènes, Diversification, Pluriactivité, Recomposition, Tourisme rural

\section{Introduction}

Depuis 1'indépendance slovène en 1991 et l'abrogation de la loi sur la propriété foncière (qui limitait les surfaces maximales autorisées des fermes individuelles), la réorganisation de l'agriculture se manifeste par une plus grande spécialisation des régions les plus productives. L'effondrement productif de l'agriculture dans les régions alpines et la forte contraction des actifs agricoles en 15 ans (-60\%) engendrent des problèmes de déclin démographique et de dégradation des prestations de services, des pertes d'emplois et de revenus. Dans ces conditions, il importait d'apprécier s'il était possible d'exploiter pour les agriculteurs, l'agritourisme comme stratégie économique de survie en tirant parti du regain d'intérêt pour la campagne, de son mode de vie traditionnel et de son patrimoine. Entre abandon de l'activité agricole et intensification de la production, la diversification vers l'agritourisme apparait comme une alternative potentielle. Qu'en est -il vraiment ? L'analyse détaillée des revenus et des structures des ménages paysans en situation d'abandon, de diversification ou d'intensification (1991-2005), accompagnée d'une série d'entretiens (2005) permet d'établir les conditions de réussite ou d'échec de l'agritourisme montagnard slovène [Barthes, 2007] et d'aborder une réflexion sur le choix effectif des ménages dans leur reconversion fonctionnelle. A travers l'exposé des trajectoires paysannes vers l'agritourisme subi ou voulu, et la place réelle de l'agritourisme dans l'économie agricole, la question de la recomposition des espaces ruraux de montagne est abordée. 


\section{1) La diversification économique vers l'agritourisme : une alternative pour les fermes et les ménages paysans?}

Le questionnement de la diversification vers l'agritourisme comme alternative potentielle à l'intensification ou à l'abandon de l'exploitation s'est effectué par le traitement des données sur la structure des ménages paysans et des exploitations agricoles, et l'évaluation des revenus des exploitants propriétaires en 1991, toujours résidants dans les fermes en 2005. Dans un second temps, les données sont rapportées au type d'espace. L'analyse des stratégies des ménages paysans s'appuie sur l'exploitation des données des recensements agricoles de 1991 et 2000, les enquêtes sur les ménages de l'office statistique national slovène de 1991, 1995, 2000 , et 2005, ainsi que celles concernant les ménages agricoles du conseil des métiers agricoles en 1991 et 2005. Les revenus ont été estimés par l'intermédiaire des données parcellaires de l'imposition 2005, complétées par les enquêtes de terrain. Il s'agit donc essentiellement d'une estimation permettant d'obtenir une échelle de comparaison. Les observations de terrain menées en 2005 ont été réalisées sur un échantillon de 363 ménages agricoles exploitants - propriétaires en 1991 et dont un membre au moins demeure toujours dans la même ferme en 2005. Cette catégorie représente environ la moitié des exploitants montagnards. La répartition géographique des ménages enquêtés s'est opérée en tenant compte de différents critères ventilés comme suit : communes de Cerkno ${ }^{1}$, Gorenja vasPoljane, Solcava, Jesersko en moyenne montagne et haute montagne enclavées ${ }^{2}$ présentant peu d'infrastructures ( $24 \%$ de l'échantillon), communes de Kranj et Naklo à proximité d'un bassin d'emploi urbain ( $28 \%$ de l'échantillon), communes dans lesquelles le flux annuel est supérieur à 20000 touristes: Bled, Bohinnj, Kranjka Gora, Radovljica (31\% de l'échantillon), zone frontalière d'intensification agricole (mais diminution du nombre d'agriculteurs) $(2 \% \mathrm{de}$ l'échantillon), plaines alpines viticoles ( $3 \%$ de l'échantillon).

\section{A) Des situations matérielles et humaines des fermes déterminant leurs trajectoires agricoles}

Afin d'éviter l'abandon de la ferme, la diversification de l'activité et l'agrandissement pour conserver l'activité exclusive et compétitive, sont les deux alternatives possibles dans le nouveau contexte concurrentiel. En effet, entre 1991 et 2000, la surface agricole utilisée moyenne par exploitation en Slovénie a augmenté de $106 \%$, tandis que le nombre d'exploitations a diminué de moitié. La tendance s'est largement poursuivie depuis. Or il apparaît que les choix stratégiques des agriculteurs sont essentiellement dictés par des facteurs objectifs qui déterminent la trajectoire post indépendance de la ferme, laissant alors peu de place aux choix individuels. Ainsi l'abandon survient généralement en priorité dans les fermes de petite taille, ne pouvant survivre dans le nouveau contexte concurrentiel, ce qui est plus fréquent en montagne qu'en plaine et qui sont en moyenne trois fois plus petites qu'au niveau national. Le ménage est généralement constitué d'un faible nombre de personnes ( 2 dans les fermes en situation d'abandon contre 4 en moyenne dans l'ensemble des familles agricoles de montagne), soit que la descendance n'existe pas, soit qu'elle ait émigré vers la ville ou à l'étranger. L'âge de ces ménages est très élevé, de presque 15 ans supérieur à la moyenne des ménages agricoles montagnards.

\footnotetext{
$1 \quad$ Carte de localisation des communes en annexe

$2 \quad$ La haute et la moyenne montagne sont définies précisément par Kladnik D. [1985]. Une analyse multicritères, intégrants des contraintes physiques, sociales, démographique et d'accessibilité a permis une cartographie précise, dont nous avons repris les principaux contours Barthes A. [2004]. Ces derniers diffèrent sensiblement des périmètres de la convention alpine, mais ont semblé plus propices à l'élaboration de notre réflexion, d'autant que celle-ci intègre quelques communes tests représentatives.
} 
De la même manière, la conservation de l'activité agricole exclusive n'a été possible qu'à des conditions bien précises qui impliquent de pouvoir potentiellement intensifier la production et augmenter la surface des exploitations. Ainsi les exploitations de montagne, dont les revenus sont restés exclusivement d'origine agricole se sont agrandies de $122 \%$ entre 1991 et 2005 , et l'intensification du travail n'a été efficace que pour les ménages de taille réduite possédant un fort capital. Les possibilités d'investissements supplémentaires nécessaires à cette adaptation ont parfois été fournies par un membre de la famille ayant préalablement émigré à l'étranger. Les vergers et les vignobles sont les seuls domaines dans lesquels il a été possible de garder une exploitation relativement extensive sans abandonner l'activité, car la plus-value des produits est plus conséquente. Mais il s'agit souvent de cultures de plaines alpines et collines qui présentent souvent des caractéristiques quasi-périurbaines.

Enfin, la diversification requiert aussi des conditions spécifiques. Ce sont en général des familles élargies dont les actifs soutiennent financièrement un nombre significatif de membres du foyer en âge de travailler. Le taux de dépendance est alors de 0,55 , ce qui signifie que seule une personne ${ }^{3}$ sur deux travaille (contre trois sur quatre en moyenne dans les foyers agricoles, avec un taux de dépendance de 0,70).

Les ménages à revenus mixtes, issus de l'agriculture et du tourisme, se trouvent par conséquent dans une position dans laquelle les potentialités de diversification des revenus ne sont pas liées tant aux opportunités et à la demande externe mais plutôt poussées par la nécessité et surtout l'impossibilité de conserver l'activité exclusive. Le choix, s'il peut être parfois et même souvent assumé pleinement, n'en est pas moins dans la plupart des cas un choix dicté par la contrainte et l'obligation de faire face au nouveau contexte concurrentiel. Les limites de l'offre du marché de l'emploi, dans un contexte de baisse des revenus agricoles et de nécessité de trouver un revenu complémentaire, obligent à trouver des solutions endogènes, utilisant les ressources propres du patrimoine familial. Signe des temps, le travail féminin décline d'ailleurs fortement depuis l'indépendance, vécu plutôt comme une contrainte qu'un choix [Verbole,1997], poussant alors particulièrement les femmes à une réflexion et un rôle premier dans la structuration de la pluriactivité.

L'exemple de l'unique ferme touristique de Zakojka (Nord de Cerkno), est à ce titre significatif. Proche du Porezen, point culminant du massif Škofjeloško Hribovje à 1632 mètres, la ferme accueille quelques skieurs en hiver sur les pentes du Črni Vrh $(1291 \mathrm{~m})$ et de nombreux randonneurs en été sur les sentiers balisés. En pluriactivité depuis 1992, la décision d'accueillir les touristes fait suite à une très forte chute des revenus de l'élevage et l'arrêt de cette activité devenue peu rentable. Le ménage gestionnaire de la ferme comprend 10 personnes dont 6 adultes qui travaillent tous à l'accueil des touristes. Quatre d'entre elles ont un travail salarié temporaire ou permanent. Employée de banque de 6 heures à 14 heures, Marjan est aide cuisinière et serveuse de 15 à 22 heures. Ses frères et beaux-frères, Aleksandar, Dimitrij, et Daniel, travaillent le matin dans les usines de palettes et autres pièces détachées en bois de sous-traitance vers 1'Allemagne (il leur faut près de 45 minutes pour se rendre à leur travail) et s'occupent, l'après-midi de la production fermière. Le père se consacre exclusivement de l'animation touristique et propose diverses prestations allant de l'équitation à l'accompagnement en ski de fond. Il a même installé un téléski dans le champ voisin. La mère s'emploie des repas et des produits de transformation de la ferme. Les productions fermières servent à nourrir exclusivement la famille et les hôtes. Le tourisme est le seul débouché de la production et actuellement la solution unique de commercialisation. Si la famille vit confortablement, la situation est parfois tendue quand l'un ou l'autre des enfants est en rupture d'emploi. 
Ainsi, les facteurs objectifs tels que le nombre de personnes disponibles pour travailler dans le ménage, nombre de personnes à charge, membres de la famille travaillant à l'extérieur ou à l'étranger, le capital disponible, la taille de l'exploitation, sont les facteurs déterminants des choix de diversification.

\section{B) Une influence de la proximité des centres urbains et touristiques}

Les facteurs géographiques jouent aussi pleinement dans le choix de diversification de la ferme vers l'activité touristique. L'abandon des exploitations est d'autant plus fort que l'on se trouve à proximité d'une ville. Elle atteint $58 \%$ dans les communes urbaines (villes alpines de plus de 20000 hab.) 49\% dans les villes moyennes (entre 10000 et 20000 hab.) et 41\% dans les plaines alpines soumises à une forte dynamique périurbaine contre $36 \%$ en moyenne. Par contre les exploitations restantes sont alors plus nombreuses à conserver une activité agricole exclusive, environ $40 \%$ des fermes encore en activité (contre $31 \%$ sur 1'ensemble de la zone). Ce sont aussi ces fermes qui ont les revenus les plus élevés. Tout se passe comme si la proximité de la ville opérait un effet sélectif accru sur les fermes qui n'ont alors pas d'autres possibilités que de s'agrandir ou intensifier la productivité pour rester compétitives ou bien disparaître, l'appel de l'emploi urbain favorisant le départ des jeunes. La proximité des frontières, et l'accès rapide à une ville autrichienne ou italienne tendent vers le même résultat. La proximité d'un marché de consommation n'a pas favorisé l'émergence de la diversification vers le tourisme

Figure $n^{\circ} 1$ : évolution 1991-2005 de l'activité et des revenus agricoles des communes urbaines de montagne

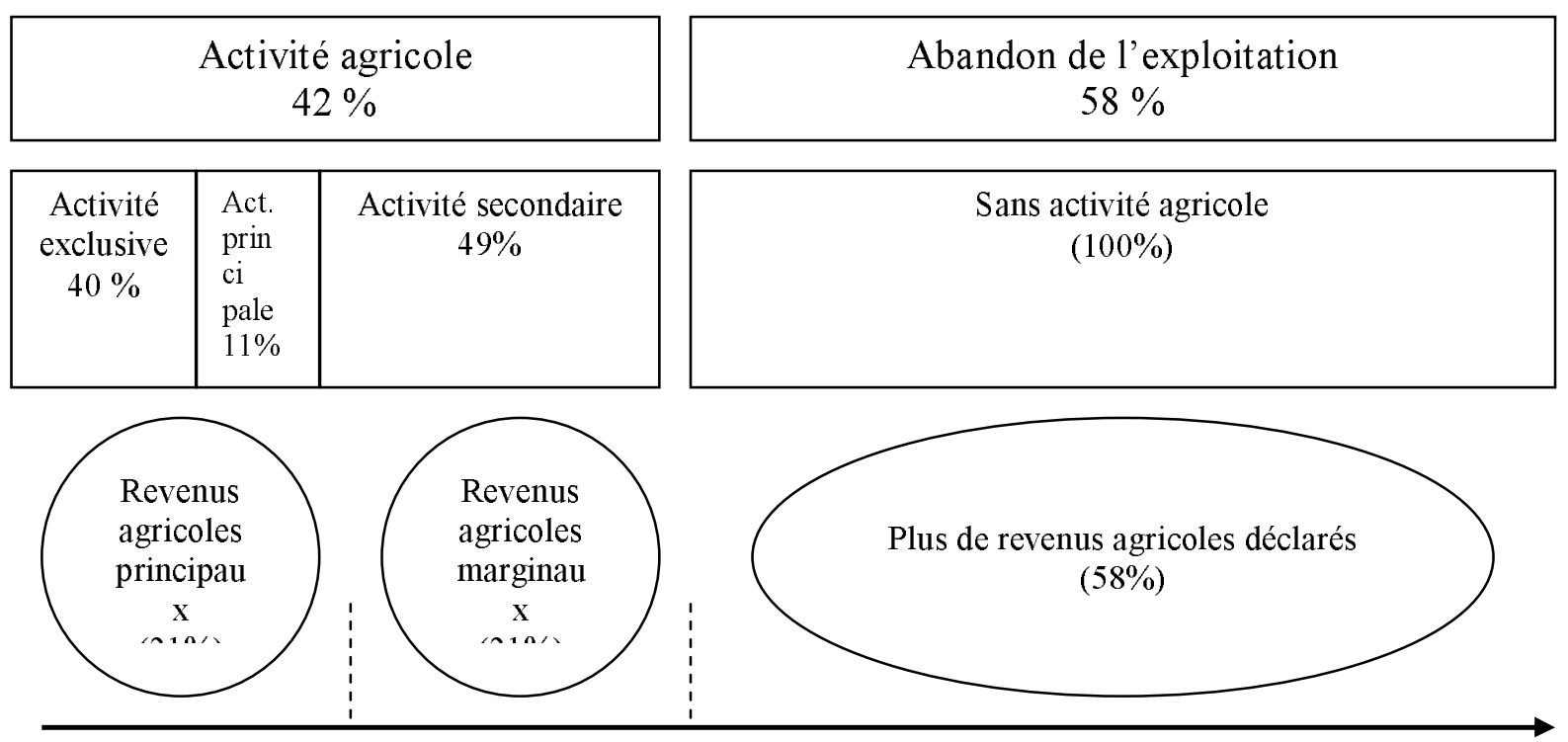

Vers un abandon de l'activité agricole à proximité des villes de montagne

Sources : calculs de l'auteur, d'après les recensements agricoles, les enquêtes sur les ménages et ménages agricoles 1991, 1995, 2000, et 2005

La proximité des stations touristiques ne semble pas un facteur favorisant le maintien de l'activité agricole. L'abandon des exploitations concerne plus de la moitié des effectifs. Le tourisme semble à double tranchant. Il participe à la spécialisation des territoires et diminue 
donc sur place les potentialités de survie de l'exploitation agricole. En même temps, il fournit les conditions nécessaires à la constitution d'exploitations dans lesquelles une part des revenus provient du tourisme (à proximité des stations touristiques, $61 \%$ des exploitations considèrent leur revenu agricole comme secondaire) seule possibilité de conserver une activité agricole. Travailler et résider dans le Parc de Triglav présente un atout supplémentaire dans la conservation de 1'activité agricole en zone touristique [Barthes, 2005]. Le taux d'abandon des exploitations est sensiblement inférieur à celui d'autres stations touristiques $(43 \%$ contre $51 \%$ ) mais reste sensiblement supérieur à la moyenne alpine (36\%). Le Parc du Triglav apparaît donc comme modérateur de l'abandon des exploitations mais il favorise en même temps la mise en tourisme, facteur d'abandon des fermes.

\section{Figure $n^{\circ} 2$ : évolution 1991-2005 de l'activité et des revenus agricoles des communes touristiques de montagne}

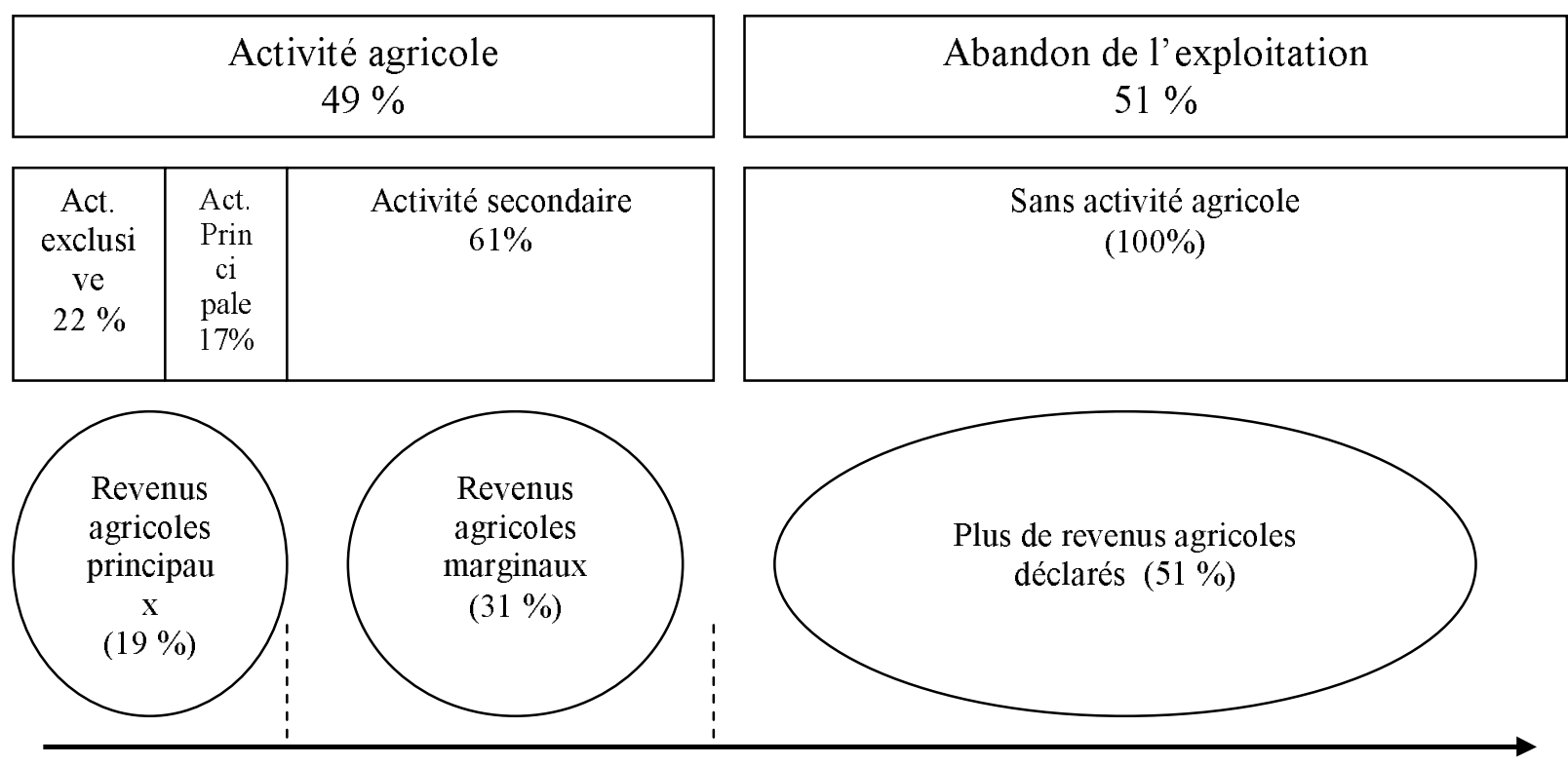

Vers une marginalisation des revenus agricoles

Sources : calculs de 1'auteur, d'après les recensements agricoles, les enquêtes sur les ménages et ménages agricoles 1991, 1995, 2000, et 2005

Paradoxalement, la moyenne montagne peu touristique mais présentant des conditions topographiques et édaphiques favorables constitue la zone géographique qui parait la plus propice aux agriculteurs. Le taux d'abandon des fermes de montagnes y est de $23 \%$, soit $13 \%$ inférieur à la moyenne, et le revenu agricole exclusif ou principal représente $51 \%$ des fermes encore en activité.

Figure $n^{\circ} 3$ : évolution 1991-2005 de l'activité et des revenus agricoles des communes de moyenne montagne 


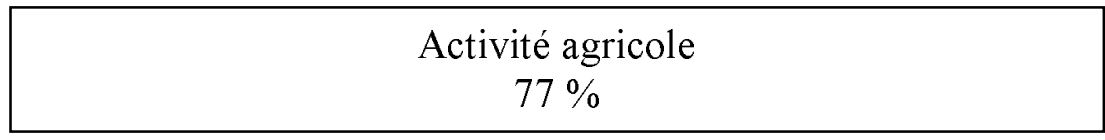

\begin{tabular}{|c|c|c|}
\hline Activité exclusive & Activité & Activité secondaire \\
$36 \%$ & princi & $49 \%$ \\
& pale & \\
& $14 \%$ & \\
\hline
\end{tabular}
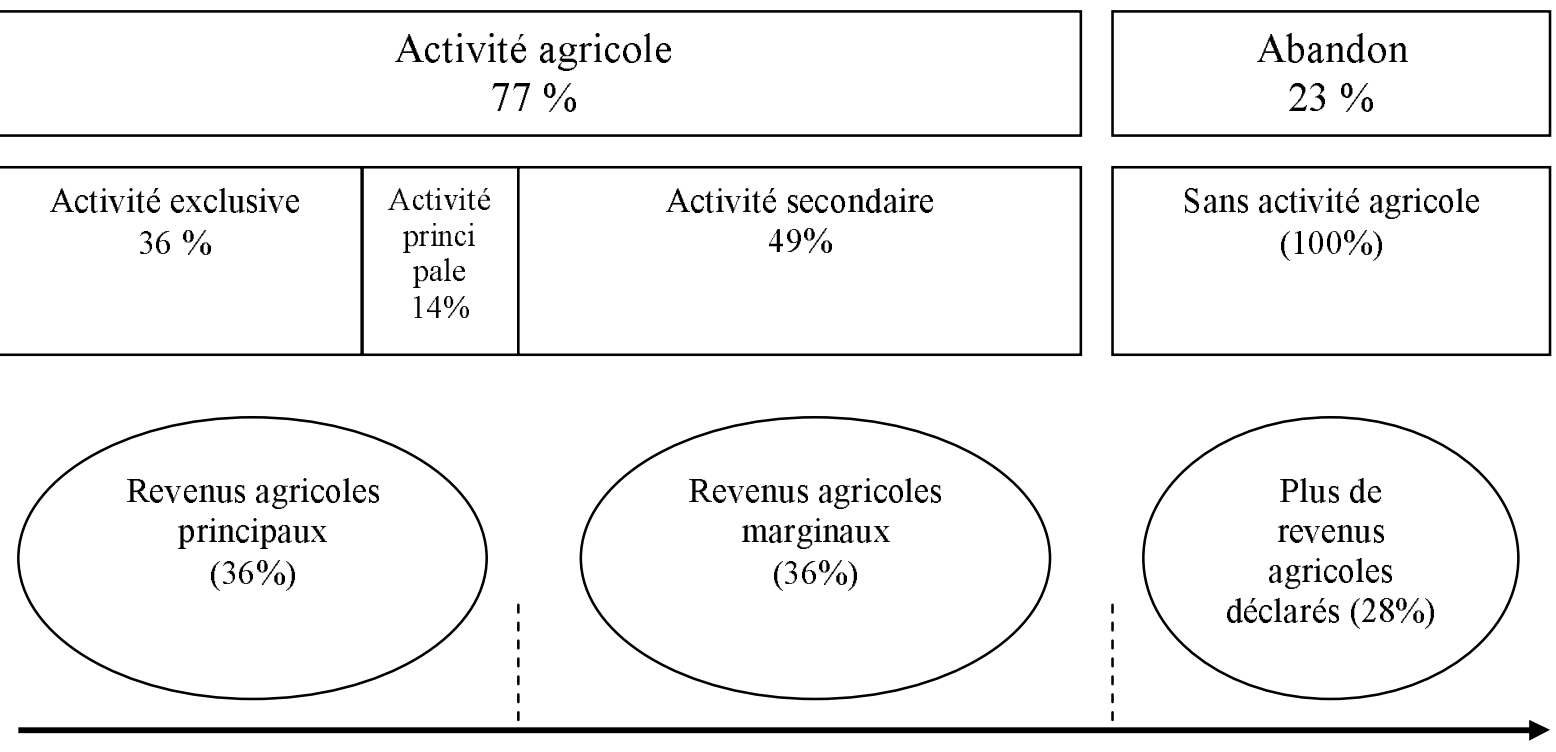

Vers une marginalisation des revenus agricoles

Sources: calculs de l'auteur, d'après les recensements agricoles, les enquêtes sur les ménages et ménages agricoles 1991, 1995, 2000, et 2005

Il est difficile de faire la part des facteurs favorisant le maintien agricole relatif en moyenne montagne. La question est posée sur l'opportunité que représente l'agritourisme dans la conservation de l'emploi -agricole ou non- in situ. Bien que ce questionnement ne se pose pas exclusivement de ces zones, il trouve une acuité particulière du fait de l'extrême dispersion de l'offre agritouristique très caractéristique en moyenne montagne. Le touriste trouve des auberges nombreuses et parsemées dans les petits villages de moyenne montagne. Par exemple, dans le massif de Škofjeloško Hribovje au nord de Cerkno, un hameau d'une cinquantaine de personnes, Zakojka, très difficile d'accès, au moins 30 minutes par des routes très tourmentées, dispose néanmoins de deux gîtes et d'une ferme auberge. Une maison sur trois est donc susceptible d'accueillir un hôte. Dans le même ordre d'idée, on soulignera l'importance de la restauration rurale, avec au moins une auberge par hameau en montagne.

\section{C) Des revenus plus faibles pour les ménages dont l'activité s'est diversifiée vers le tourisme}

Le choix effectif des ménages vers la diversification est remis en cause par l'objectivation de la situation pré et post-indépendance de la ferme, mais la structure des revenus apporte quelques éléments supplémentaires allant dans le même sens. En effet, les revenus des ménages qui ont pu ou su garder une activité agricole exclusive sont supérieurs aux revenus des ménages dont l'activité s'est diversifiée (Fig.4). Les familles sont plus petites, les fermes plus grandes, et les revenus proviennent pour le tiers environ des subventions. Même si l'écart est moins marqué, les revenus des ménages dont la ferme a été abandonnée sont supérieurs, et près de $60 \%$ d'entre eux proviennent d'un emploi externe. La potentialité de trouver un emploi est donc financièrement préférable à la pluriactivité.

Figure $n^{\circ}$ 4: estimation des revenus 2005 des ménages agricoles exploitantspropriétaires en 1991 


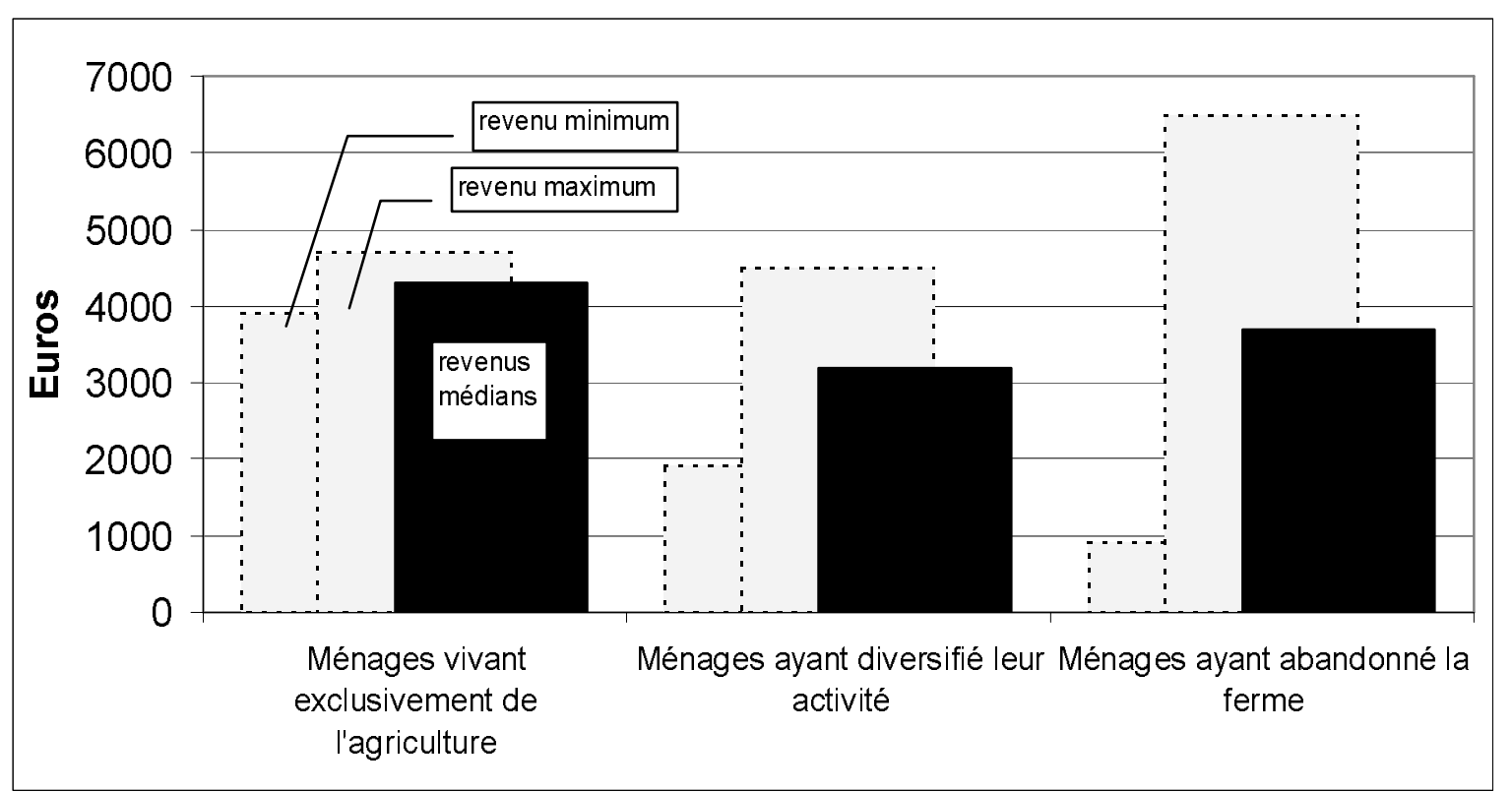

Sources : estimation d'après enquêtes sur les ménages et ménages agricoles 2005, conseil des métiers agricoles, impôts 2005, enquête de terrain 2005. Il s'agit donc essentiellement d'une estimation permettant d'obtenir une échelle de comparaison.

\section{2) Agriculture versus tourisme rural, quelle ruralité se construit à travers les restructurations économiques?}

Dans un contexte de très forte érosion de l'activité agricole, l'agritourisme est souvent considéré comme une stratégie de développement alternative pour la régénération économique et sociale des campagnes [Hjalager, 1996] [Verbole, 1997] [Rátz a Puczkö 1998] [Bavec et al., 2002] [Oostindie, Van der Ploeg, Renting, 2002]. Du point de vue du développement régional, le programme touristique slovène du Ministère de l'économie de 2002 s'appuie beaucoup sur le développement du tourisme rural qui, de par son intégration géographique potentielle à un territoire très peu densément peuplé, présente un attrait complémentaire mais fondamental au tourisme urbain et des grandes stations thermales ou de ski.

L'analyse de la situation des ménages paysans d'une part et l'histoire locale de l'agritourisme dans le contexte global permet d'avancer une hypothèse sur la place réelle au delà des discours dominants, que tient le tourisme rural d'une part à l'échelle des ménages, d'autre part à celles des espaces montagnards

\section{A) Une dispersion géographique réussie mais une faible emprise géographique de l'agritourisme}

Le tourisme rural a commencé à se développer dans les années 90 après 1'application de la loi sur l'hôtellerie qui fixe les réglementations de bases et définit les normes pour les différentes formes de fermes touristiques potentielles. En 2006, il est dénombré 482 fermes agritouristiques. Parmi elles, seules 189 proposent des hébergements, la majeure partie se concentre prioritairement sur la restauration et la gastronomie. 190 fermes proposent des activités complémentaires telles que l'accompagnement en randonnées, le ski, le cheval ou le vélo. 74 fermes participent à un réseau de tourisme liée au vignoble et au vin. Enfin, il est dénombré 27 « osmic » (fermes qui ont la permission pendant huit jours par an de vendre 
leurs produits sans payer de taxe). La capacité totale d'accueil de ces fermes est de 22284 couverts et 2595 lits par jours ouvrables. La taille des structures d'accueil est très variable : en moyenne d'une capacité de 13 lits. La plus petite possède deux chambres doubles, et la plus grandes 31 places. $70 \%$ des établissements qui proposent des hébergements sont aussi des restaurants. La durée de la saison touristique diffère d'un lieu à l'autre, si bien qu'il est très difficile de dénombrer la disponibilité réelle en intersaison. Le niveau de confort des établissements est déterminé grâce aux « étoiles » (de 1 à 3 ) ou aux « pommes » (de 1 à 4). Les établissements les plus luxueux se trouvent essentiellement à proximité des grandes stations touristiques ou des villes, ou dans la principale vallée alpine reliant le Ljubljana au Triglav. On note ici l'absence de fermes agritouristiques de confort inférieur à moyen dans ces zones qui côtoient le tourisme de masse, comme si la concurrence plus rude ne permettait pas leur installation. Au final, la répartition géographique des fermes est très homogène sur tout le territoire de montagne, tandis que la dispersion est la caractéristique principale de l'agritourisme alpin. Tout au plus y a t'il une faible augmentation du nombre de restaurants dans les vallées et à proximité des frontières italiennes et autrichiennes. Le tourisme lié au vignoble se localise dans les Préalpes tandis que l'équitourisme prospère plus volontiers en moyenne et haute montagne.

L'enquête auprès des ménages montre qu'au final la reconversion touristique des fermes n'a pas concerné de nombreux établissements. Elle représente aujourd'hui un peu moins de 5\% de l'ensemble des fermes actuelles (soit moins de 3\% des fermes en activité en 1991) car l'obtention du label et les normes d'hygiène sont strictes et parfois incompatibles avec certaines habitudes culturelles. Les quelques parcours typiques identifiés font suite à des arrêts brutaux de l'élevage fermier, de bovins par exemple, dans le contexte de débandade généralisée post- indépendance dans le secteur. La ferme réinvestit ses compétences en matière d'élevage par l'introduction d'animaux destinés au tourisme. Il s'agit d'une conversion en ferme équestre ou de l'élevage de gibier pour la gastronomie. Les animaux deviennent alors produits d'appel pour le tourisme. Le label «agriculture biologique» est très fréquemment obtenu, car les pratiques culturales anciennes utilisaient des méthodes proches du cahier des charges demandé et constitue un atout touristique non négligeable.

C'est dire la faible emprise que représente la diversification des fermes vers le tourisme, surtout comparée au nombre de fermes en rupture d'activité sur la période 1991-2005: les emplois crées par l'activité touristique des 482 fermes ne remplacent pas ceux perdus par les milliers d'exploitations ruinées. En montagne, le ratio du nombre de fermes agritouristiques nouvellement crées sur le nombre de fermes ruinées est encore plus bas (moins de $2 \%$ ), dans une zone où de surcroît les emplois tertiaires viennent beaucoup moins remplacer les emplois perdus dans les secteurs primaires et secondaires (fig.5).

Figure $n^{\circ}$ 5: évolution des secteurs d'emplois en montagne et bilan des emplois perdus in situ (en \%) 


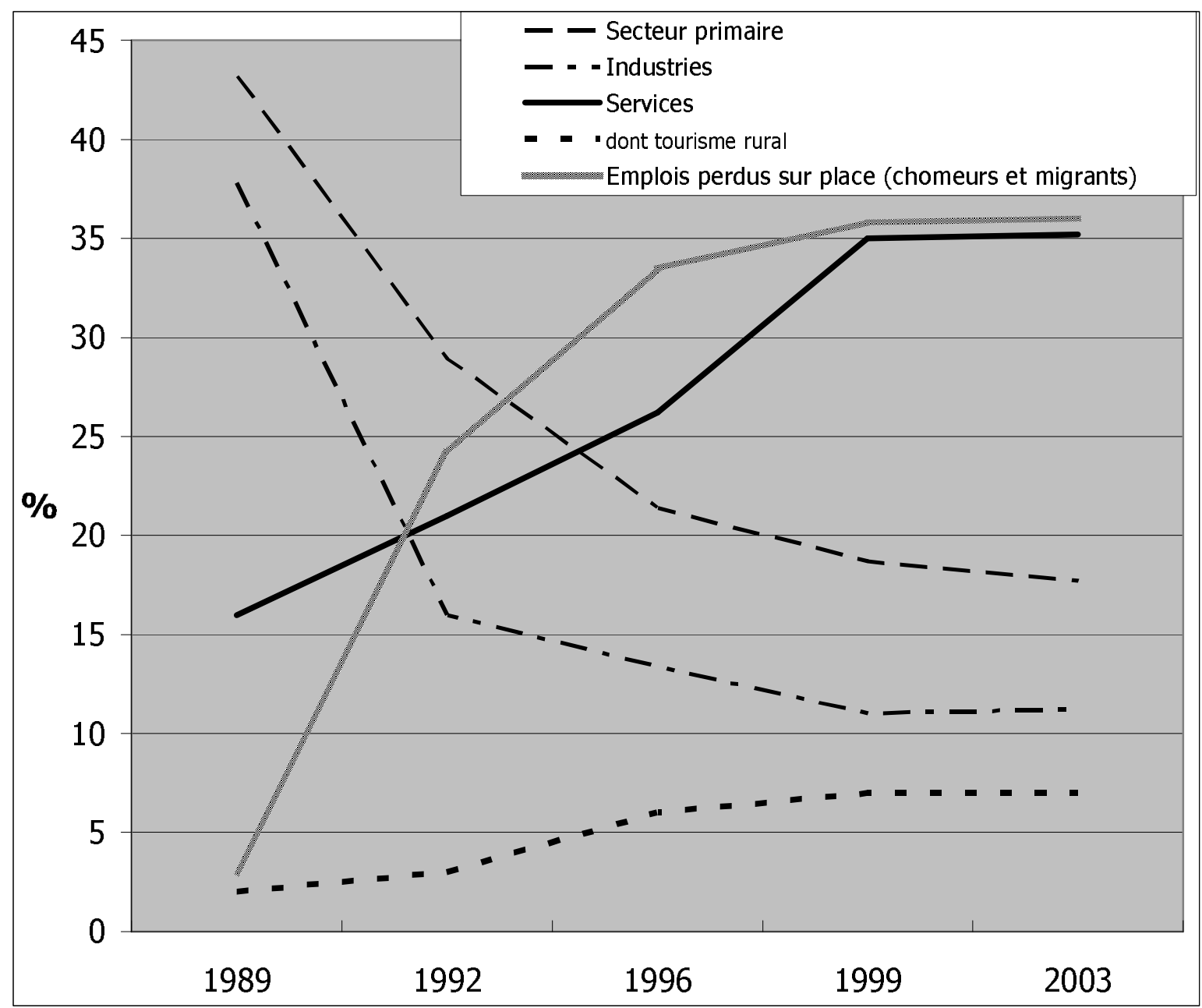

Sources : office Statistique National Slovène

Mais le tourisme rural n'est pas l'apanage exclusif des fermes en voie de diversification. Un certain nombre d'installations nouvelles de chambres et tables d'hôtes a émergé à la suite d'un rachat à bas prix des terres et bâtiments restitués après 1991 à des propriétaires ayant émigré dans les décennies précédentes, par exemple en Allemagne ou aux Etats-Unis. Ces derniers cèdent à leur famille restée au pays le foncier en échange d'une gratuité d'accueil en vacances ou pour échapper aux contraintes telles que le service militaire. Très souvent, il s'agit d'une activité nouvelle qui ne fait pas suite à la reconversion d'une ferme. Les établissements de tourisme rural sont quatre fois plus nombreux que les fermes agritouristiques, ce qui constitue également un pourcentage d'emplois assez faible. Le seuil semble atteint puisqu'il n'y a plus aujourd'hui de croissance réelle du nombre d'établissements.

En dépit de nombreux investissements aidés par les subventions, l'agritourisme ne semble pas apporter les revenus complémentaires attendus (Fig. 5) et ne présente pas une solution prépondérante de la conservation de l'activité en moyenne montagne [Verbole, 1997], comme le montre la structure des revenus des ménages. Parmi les freins au développement agritouristique, est soulignée la complexité de sa mise en œuvre. Elle demande l'implication de toute la famille et des compétences multiples, linguistiques, gastronomiques. L'affichage de spécialités culinaires est un appel pour les touristes. De nombreuses fermes proposent ainsi de la cuisine de viande de gibier, dont l'élevage est réalisé sur l'exploitation: daims, chevreuils, etc. De nombreux produits transformés sur place : jus, alcools, vins, fromages, 
confitures, miels, charcuteries, pain... Les compétences des familles doivent s'étendre aux activités sportives afin de proposer des cours d'équitation, de ski, des randonnées. Les capacités communicationnelles à s'insérer dans un réseau local par exemple avec les moniteurs de ski pour augmenter l'offre touristique sont essentielles, comme la mise en oeuvre des politiques de promotion via les réseaux associatifs ou via l'Internet. Il est important également de posséder des capacités au bricolage,et à la possibilité d'obtenir un second emploi, etc. La normalisation des fermes agritouristiques permet les prémisses d'une organisation collective et une meilleure lisibilité de l'offre. Par contre elle impose des investissements élevés aux candidats à l'agritourisme créant par la même une sélection importante entre les agriculteurs. Une assistance financière et technique est parfois proposée, mais compense rarement les incertitudes du marché, la concurrence du tourisme de masse, les lacunes des compétences nécessaires pour aborder cette nouvelle activité complexe.

Ainsi, l'agritourisme influe peu sur l'activité agricole mais sur la conservation de la ferme par le ménage qui opère sa conversion fonctionnelle. Les productions deviennent tout à fait marginales, ne sont plus vendues mais servies aux hôtes, tandis que l'emploi se conserve ponctuellement. L'ensemble des emplois dégagés est très inférieur aux emplois perdus postindépendance. Ainsi, en dépit d'un discours sur les potentialités touristiques en zones rurales qui se veut rassurant, il parait déraisonnable de considérer l'agritourisme comme solution de revitalisation des montagnes slovènes. Tout au plus, cela permet-il le maintien d'une activité productive minimale sur place, ce qui est déjà fondamental. Mais les questions d'échelles et le poids économique des activités ne sont pas les seuls facteurs à prendre en compte.

\section{B) Une organisation en réseau : vers un décloisonnement des agriculteurs}

L'organisation en réseau des fermes agritouristiques est déjà bien entamée. Les fermes touristiques slovènes se sont dotées d'une association, «Združenje turistične kmetij Slovenije » fondée en 1997, dont la fonction principale est leur promotion. Elle regroupe la majeure partie des services d'hébergements fermiers, puisque les informations de la brochure annuelle 2006 font état de 2000 lits disponibles (sur 2595 au total). En ce qui concerne la restauration seule la moitié des fermes fait partie du réseau principal avec 11000 couverts journaliers proposés. D'un point de vue de la promotion touristique des fermes, d'après une enquête permanente réalisée auprès des usagers, 50\% des informations proviennent des différents catalogues de l'association, qui devient donc un maillon essentiel de l'organisation de 1'offre agritouristique fermière. $35 \%$ des informations proviennent des sites Internet des fermes elles-mêmes, lesquels sont centralisés également sur le site Internet de l'office national $\mathrm{du}$ tourisme slovène. Là encore, 1'organisation en réseau prime, puisque pour $76 \%$ des internautes, l'entrée première a été constituée par le site centralisateur. Seulement $5 \%$ des touristes obtiennent ce genre d'informations dans les agences touristiques. Les autres, viennent suite à un contact antérieur ou grâce au bouche à oreille. Grâce à cette organisation, de plus en plus d'usagers proviennent de l'étranger. Les Autrichiens et les Italiens deviennent les premières nationalités étrangères à utiliser les offres du tourisme rural slovène.

\section{C) Le tourisme rural, un facteur parmi d'autres d'une reconversion fonctionnelle des montagnes slovènes}

La genèse d'une nouvelle forme d'organisation en réseau, une lisibilité accrue et une ouverture sur l'international constituent un facteur culturel non négligeable dans les potentialités de reconversion fonctionnelle des espaces montagnards slovènes. Ainsi le tourisme rural, s'il joue un rôle mineur dans la restructuration économique des campagnes en 
forte perte d'emplois agricoles, joue un rôle structurant important permettant la potentialité accrue d'une reconversion de ces mêmes espaces. Notamment, il sert de tremplin pour l'achat de résidences secondaires et de la reconversion vers un espace de villégiature, au même titre que l'extension périurbaine de la capitale en vallée le long de l'axe Ljubljana-Kranj. La forte chute du nombre d'emploi en montagne, génère également une croissance des mobilités journalières, qui participent aussi à la transformation de l'espace rural en un espace intermédiaire de villégiature dont la fonction productive diminue considérablement, avec une interpénétration accrue de la ville et de la campagne.

\section{Conclusion}

I1 est frappant de constater l'extrême similitude des choix privés dans des situations équivalentes. Tout se passe comme si l'initiative individuelle n'avait au fond que peu d'impact. Parfois, les compétences font la différence, mais la marge de manœuvre reste très courte, à tel point qu'il est presque possible d'évaluer l'évolution probable des fermes dans la décennie à venir. Il est frappant également de constater que les revenus restent supérieurs quand l'activité agricole est conservée. Les choix d'abandon d'activité ou de diversification sont donc prioritairement dictés par la contrainte parfois non consciente ou l'impossibilité de s'agrandir. Le tourisme ne présente que peu d'opportunité d'accroître les revenus pour les ménages paysans, en dépit de l'image valorisante qu'il véhicule.

Dans ce cadre, le tourisme rural ne présente évidemment pas une solution de revitalisation de la campagne, tant son poids économique est faible et son exercice difficile pour les ménages agricoles. S'il constitue une potentialité de reconversion des fermes mises en difficultés dans le nouveau contexte concurrentiel, il concerne une très faible part des ménages, et n'offre pas les dividendes espérés, tant la mise en oeuvre est complexe. Néanmoins, la structuration d'un tourisme rural favorise l'ouverture de l'espace peu accessible. Il favorise sa lisibilité et l'interconnaissance culturelle et concourt à la reconversion fonctionnelle des espaces ruraux. Ils agissent comme des facilitateurs de la reconversion d'espaces essentiellement productifs en lieux de vie, même si cela se fait globalement plutôt sous la contrainte économique pour les populations rurales.

\section{Annexe: Carte de localisation des communes du périmètre alpin}

\section{Bibliographie}

Barbi A. [1994], " Agricultural change in rural society and the state in Central and Eastern Europe ». In D. Symes and A.J. Jansen (eds), Agricultural Restructuring and Rural Change in Europe, Sociological Studies 37, pp. 296-309, Wageningen Agricultural University.

Barthes A. [2004], « Crise et réorganisation des Alpes slovènes depuis 1'indépendance », Méditerranée n 3-4, pp 31-36

Barthes A. [2005], «Appropriation territoriale du grand site du Mont Triglav (Slovénie) et conflictualité », Méditerranée $\mathrm{n}^{\circ} 3-4, \mathrm{pp}$ 81-86

Barthes A. [2006], «Exacerbation des disparités régionales lors de la transition socio-économique dans les pays post-socialistes d'Europe de l'est : l'exemple Slovène », Revue trimestrielle d'EPO, Bruxelles, $E M, \mathrm{n}^{\circ} 74$, pp 5381 
Barthes A. [2007], «Transition socio-économique et adaptation des agriculteurs dans les Alpes Slovènes », Actes du colloque Héritages et trajectoires rurales en Europe, 6 et 7 septembre 2007, Montpellier

Bavec, M., et al. [2002], «Ekolo kmetijstvo ob vstopu Slovenije v Evropsko, unijo ». Biodar 2-3.

Bavec, M., et al., [2002], «Ekolo kmetijstvo - Slovenska prilo nost v Evropski uniji? » 17. tradicionalni posvet Kmetijske svetovalne slu'be Slovenije, Bled.

Benkovič M. [2001], « Idrijko hribovje kot primer demografsko ogroženega obmožja », Dela 17, pp138-151, Ljubljana

Benkovič M. [2005], « Razvojne možnosti naselij na depopulacijskih območjih glede na starostno sestavo gospodinjstev », Geografski vestnik 77-2, pp 81-93

Buchenrieder G., Möllers J., Heidhues F. [2003], « Ein Ländervergleich der Außerlandwirtschaflichen Beschäftigung in Macédonien and Slowenien », 43 Jarestagung der Gesellschaft für Wirschafts und Sozialwissenschaften des Landbaus vom 29.September-1 Oktober 2003, Stuttgart.

Cerne A. [2003], « Regional development perspectives of Slovenia » Dela 19, pp107-121, Ljubljana

Hjalager, A. M. [1996], « Agricultural Diversification into Tourism », Tourism Management 17.

Kulovec, M. [2002], «Dopolnilne dejavnosti na kmetijah. Sodobno kmetijstvo 35.

Kladnik D. [1985], « Problematika zemljiske strukture v Sloveniji. Elaborat, 192 strani.», Institut za geografijo Univerze Edvarda Kardelja v Lubljana,

Klemenčič M. [1995], « La Slovénie subalpine, entre renaissance et déclin », Revue de géographie alpine, $\mathrm{n}^{\circ}$, tome 83 , pp. $39-50$

Kombol, T. P. [1998], « Rural Tourism and Croatia's Islands », Paper presented at the rural tourism Management: Sustainable Options International Conference. Auchincruive.

Meze D. [1984], « Mountain farms in Slovenia », Geographica Iugoslavice, 5, pp. 47-55

Meze D. [1987], « Mountain farms in the Idrija and Cerklje regions », Geografski zbornik, 27, pp.5-67

Nilsson, A. [2002], « Staying on farms; An Ideological Background » Annals of Tourism Research 29-1.

Perpar A., Kovačic M. [2001], « Tipologija in razvojne značilnosti podeželskih območij v slovenji », dela 17, pp 85-99, Ljuljana

Ravbar M. [2004], « Regional development in the regional division of Slovenia », Acta geographica Slovenica, $44-1, \mathrm{pp} 7-24$

Schneider F., et Klingmair R. [2004], « Main labor market development during the transition, in enhancing job opportunities: Eastern Europe and the Former Soviet Union», Banque Mondiale 2004, pp61-105

Oostindie H., van der Ploeg, J.D., Renting, H. [2002], « Farmers' experiences with and views on rural development practices and processes: outcomes of a transnational European survey » Multifunctional Agriculture, A new paradigm for European Agriculture and Rural Development. Aldershot.

Rátz, T., Puczkö, L. [1998], « Rural Tourism and Sustainable Development » Rural Tourism Management: Sustainable Options International Conference. Auchincruive.

Verbole A. [1995], « Tourism development in the European countryside: Costs and benefits » In A. Barbi and D. Wastl-Walter (eds) Sustainable Development of Rural Areas: From Global Problems to Local Solutions. Klagenfurter Geographische Zeitung 13, 60-75. Klagenfurt.

Verbole A. [1997], « National action plan for the integration of rural women in development » rapport de la FAO 
Verbole A. [1997], « Rural tourism and sustainable development: A case study on Slovenia » In H. De Haan (ed.) Sustainable Rural Development (pp. 197-215), Aldershot: Ashgate.

Verbole A. [1998], « Rural tourism development in Slovenia: An insight into negotiation of terms of sustainable rural tourism development policy » In D. Hall and L. O'Hanlon (eds) (1998) Rural Tourism Management: Sustainable Options (pp. 577-595). Auchincruive: The Scottish Agricultural College (SAC).

Verbole A. [1999], « Negotiating Rural Tourism Development at the Local Level: A Case Study in Pišece, Slovenia » Wageningen Agricultural University, $\mathrm{PhD}$ thesis

Vrišer I. [2002], «Agricultural production in the Republic of Slovenia (according to the census of the agricultural sector 2000) », Acta Geographica Slovenica ${ }^{\circ} 42$, pp. 8-60

\section{Sources}

Statistical Yearbook [1991], [1995], [2000], [2001], [2003], [2005] Statisticni urad Republike Slovenije, Ljubljana

Census of agriculture [1991] et [2000] Statisticni urad Republike Slovenije, Ljubljana

Statistiques du conseil des métiers agricoles [1991] et [2000]

Les revenus ont été estimés par l'intermédiaire des données parcellaires de l'imposition 2005, complétées par les enquêtes de terrain. 